\title{
Technical School Gold Medal Winners
}

CIF/IFC Gold Medal winners for 1993 have been announced by the Algonquin College of Applied Arts and Technology, Cégep de l'AbitibiTémiscamingue, Cégep de Sainte-Foy and Sir Sandford Fleming College.

Algonquin College of Applied Arts and Technology, Pembroke, Ontario. John Askitis.

John Askitis was born near Johannesburg, South Africa, educated in Pretoria, and came to Ottawa in 1990. By trade John is a heavy equipment mechanic with 12 years of experience in South Africa. He entered the Forestry Technician Program at Algonquin College in order to explore opportunities to combine his trade with a new career in his great interest, forestry. The enthusiasm and thoroughness that John applies to every task assigned to him assure a successful transition to this new career.

Cégep de l'Abitibi-Témiscamingue, Rouyn-Noranda, Quebec Etienne Garant.

Etudiant en $3^{\mathrm{e}}$ année de techniques forestières au Collège de l'AbitibiTémiscamingue Etienne Garant fait partie des forestiers déterminés à bien aménager nos forêts. Sa détermination au cours de ses études lui a permis de maintenir un dossier académique remarquable, tout en travaillant à temps partiel pendant ses études. Il a su également s'impliquer activement dans les activités socioculturelles et sportives organisées par ses collègues. Au cours de ses vacances d'été, il a occupé différents postes d'entretien et de production au sein d'une importante scierie de la région, mariant ainsi la pratique à la théorie.

Cégep de Sainte-Foy, Sainte-Foy, Quebec, François Tremblay

Originaire de la région de l'Estrie, François complète ses études secondaires au Collège du Mont Sainte-Anne à Rock Forest. À l'automne 1991, il entre au Cégep de Sainte-Foy en technologie forestière. Il travaille deux étés consécutifs à l'usine de Domtar à Windsor avant de se retrouver en Abitibi, à l'été 91, sur des contrats de reboisement pour la Médéole. À l'été

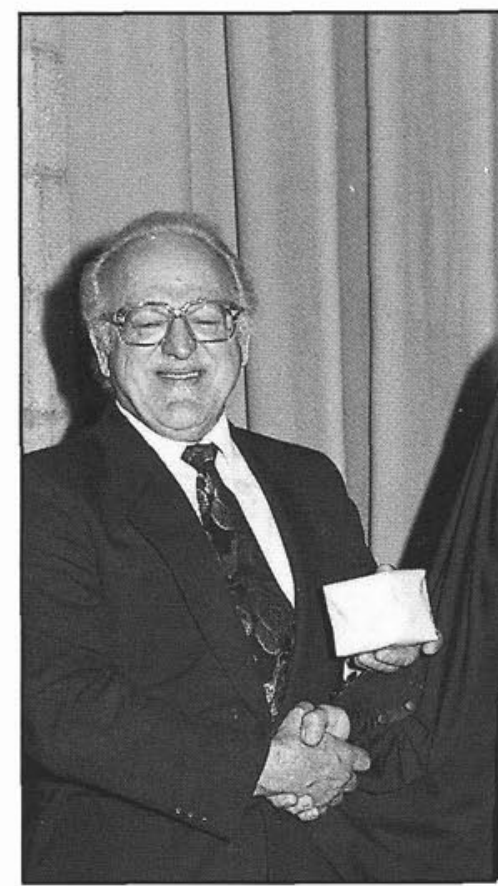

Mr. Lawrence Scales, Chair of the Algonquin Section of the Canadian Institute of Forestry, on the left, and the recipient, John Askitis, on the right.

À Gauche: P. Bernier, president de la section Orleans. A Droite: F. Tremblay
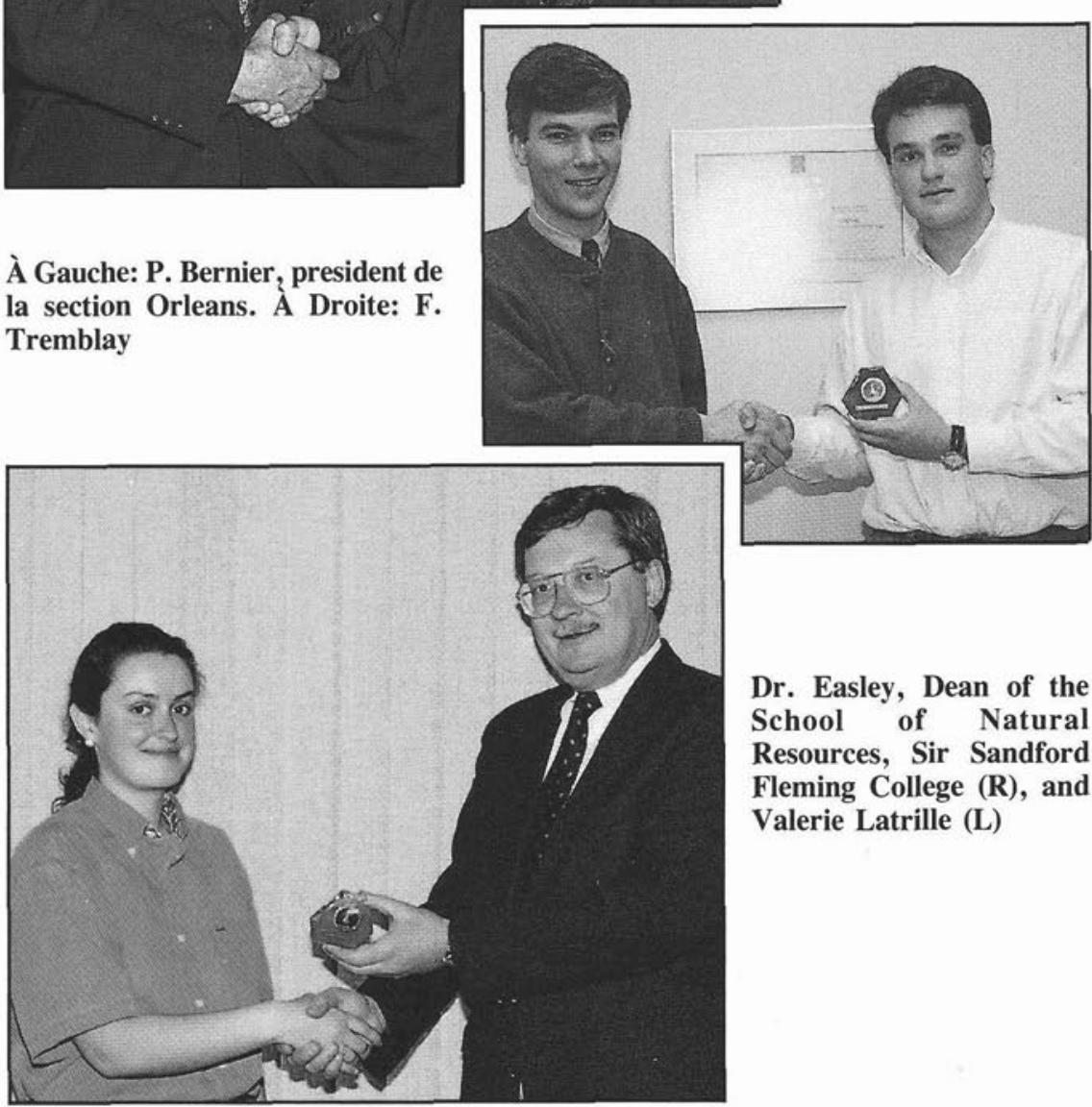

Dr. Easley, Dean of the School of Natural Resources, Sir Sandford Fleming College (R), and Valerie Latrille (L)
92, il travaille au Centre de foresterie des Laurentides où il se découvre une nouvelle passion: l'entomologie forestière. François souhaite d'ailleurs pour-suivre ses études en foresterie à l'Université et travailler plus tard dans ce domaine. Amateur de chasse et de pêche, François s'intéresse aussi fortement au camping et à tout ce qui touche le plein air en général.
Sir Sandford Fleming College, Lindsay, Ontario, Valerie Latrille. The award was presented to Valerie for outstanding scholarship, sports and citizenship recorded throughout her time spent in the Forestry Program. 